\title{
GENERAL ITERATIVE METHODS FOR NONLINEAR BOUNDARY VALUE PROBLEMS
}

\author{
RADHA SHRIDHARAN ${ }^{1}$ and RAVI P. AGARWAL ${ }^{2}$
}

(Received 22 June 1993; revised 10 September 1993)

\begin{abstract}
In this paper we shall develop existence-uniqueness as well as constructive theory for the solutions of systems of nonlinear boundary value problems when only approximations of the fundamental matrix of the associated homogeneous linear differential systems are known. To make the analysis widely applicable, all the results are proved component-wise. An illustration which dwells upon the sharpness as well as the importance of the obtained results is also presented.
\end{abstract}

\section{Introduction}

In this paper we shall consider the boundary value problem

$$
\begin{gathered}
x^{\prime}=f(t, x), \quad t \in J=[a, b], \\
g[x]=0,
\end{gathered}
$$

where $x$ and $f$ are $n$-dimensional vectors, $g$ is an operator from $C(J)$ into $R^{n}$ and $C(J)$ is the space of $n$ vector functions which are continuous on $J$. The motivation to study (1.1), (1.2) comes from the fact that it includes various practical problems, including those arising in optimal control theory [21]. In existence-uniqueness as well as constructive theory of (1.1), (1.2) the explicit form of the fundamental matrix of the associated homogeneous linear differential systems plays an important role [1-3,5,7,9,10,13-17,19-25]. However, in [11] it has been noted that in practice the explicit form of this matrix is rarely known. Therefore, to have a wider applicability of the methods it is necessary to restudy $(1.1),(1.2)$ when only approximations of the fundamental matrix are known.

'BLK 112, Tampines Street 11, \# 08-195, Singapore 1852.

${ }^{2}$ Department of Mathematics, National University of Singapore, Singapore 0511 for correspondence.

(C) Australian Mathematical Society, 1995, Serial-fee code 0334-2700/95 
The plan of this paper is as follows. In Section 2 we list some properties of square matrices which are used throughout this paper without further mention; state a contraction mapping theorem in generalized Banach spaces, and for the invertability of a linear operator which maps a generalized Banach space to another generalized Banach space provide necessary and sufficient conditions. In Section 3, we shall follow Hayashi [11] to obtain explicit representations of the solutions of the linear boundary value problems in terms of the exact as well as approximate fundamental matrices of the associated homogeneous differential systems. These explicit representations are used in Section 4 to prove the convergence of Picard's iterative methods for the boundary value problem (1.1), (1.2). The obtained results here are more general and precise than those available in [11]. Section 5 is devoted to the computational aspects of Picard's iterative schemes developed in Section 4. An example which dwells upon the importance as well as the sharpness of the obtained results is included in Section 6. All the results in this paper are proved in generalized (vector) normed spaces. The significance of such a study for systems is now well recognized from the fact that it enlarges the domain of existence and uniqueness of solutions, weakens the convergence conditions and provides sharper error estimates; for example, see $[1-8,12,18,20,25]$.

\section{Preliminaries}

Throughout this paper we shall consider the inequalities between two vectors in $R^{n}$ component-wise, but between two $n \times n$ matrices, elementwise. The following well-known properties of matrices will be used frequently.

(1) For any $n \times n$ matrix $A, \lim _{m \rightarrow \infty} A^{m}=0$ if and only if $\rho(A)<1$, where $\rho(A)$ denotes the spectral radius of $A$.

(2) For any $n \times n$ matrix $A,(I-A)^{-1}$ exists and $(I-A)^{-1}=\sum_{m=0}^{\infty} A^{m}$ if $\rho(A)<1$, where $I$ denotes the unit matrix. Also, if $A \geq 0$, then $(I-A)^{-1}$ exists and is nonnegative if and only if $\rho(A)<1$.

(3) If $0 \leq B \leq A$ and $\rho(A)<1$ then $\rho(B)<1$.

(4) (Toeplitz Lemma). For a given $n \times n$ matrix $A \geq 0$ with $\rho(A)<1$ and a sequence of vectors $\left\{d_{m}\right\}$, we define the sequence $\left\{s_{m}\right\}$, where $s_{m}=\sum_{i=0}^{m} A^{m-i} d_{i}$; $m=0,1,2, \ldots$. Then $\lim _{m \rightarrow \infty} s_{m}=0$ if and only if $d_{m} \rightarrow 0$.

THEOREM 2.1 (Contraction Mapping Theorem [1]). Let $B$ be a 'generalized (vector) Banach Space' and let $r \in R_{+}^{n}, r>0: \bar{S}\left(x_{0}, r\right)=\left\{x \in B:\left\|x-x_{0}\right\| \leq r\right\}$. Let $T$ map $\bar{S}\left(x_{0}, r\right)$ into $B$ and

(i) for all $x, y \in \bar{S}\left(x_{0}, r\right),\|T x-T y\| \leq K\|x-y\|$, where $K \geq 0$ is an $n \times n$ matrix,

(ii) $\rho(K)<1$, and $r_{0}=(I-K)^{-1}\left\|T x_{0}-x_{0}\right\| \leq r$. 
Then the following hold:

(1) $T$ has a fixed point $x^{*}$ in $\bar{S}\left(x_{0}, r_{0}\right)$,

(2) $x^{*}$ is the unique fixed point of $T$ in $\bar{S}\left(x_{0}, r\right)$,

(3) the sequence $\left\{x_{m}\right\}$ defined by $x_{m+1}=T x_{m}, m=0,1,2, \ldots$, converges to $x^{*}$ with $\left\|x^{*}-x_{m}\right\| \leq K^{m} r_{0}$,

(4) for any $x \in \bar{S}\left(x_{0}, r_{0}\right), x^{*}=\lim _{m \rightarrow \infty} T^{m} x$,

(5) any sequence $\left\{\bar{x}_{m}\right\}$ such that $\bar{x}_{m} \in \bar{S}\left(x_{m}, K^{m} r_{0}\right), m=0,1,2, \ldots$, converges to $x^{*}$.

For $x(t)=\left(x_{1}(t), \ldots, x_{n}(t)\right) \in C(J)$ we shall denote by $|x(t)|=\left(\left|x_{1}(t)\right|, \ldots\right.$, $\left.\left|x_{n}(t)\right|\right)$ and $\|x\|=\left(\sup _{t \in J}\left|x_{1}(t)\right|, \ldots, \sup _{t \in J}\left|x_{n}(t)\right|\right)$. The space $C(J)$ equipped with this norm is a generalized normed space. If $x \in R^{n}$, then obviously $x \in C(J)$, and hence $|x|=\|x\|=\left(\left|x_{1}\right|, \ldots,\left|x_{n}\right|\right)$. Let $M(J)$ denote the Banach space of all real $n \times n$ matrix valued functions $A(t)$ which are continuous on $J$ with the norm analogous to the $n$ vector functions.

For any fixed $t_{0} \in J$, let $C_{0}(J)=\left\{x \in C(J): x\left(t_{0}\right)=0\right\}$. Then, $B_{0}=C_{0}(J) \times R^{n}$ is a Banach space with the norm $\|y\|=\max (\|u\|,\|e\|)$ for $y=(u, e) \in B_{0}$. As usual $L\left(B_{1}, B_{2}\right)$ denotes the set of all bounded linear operators from the Banach space $B_{1}$ into the Banach space $B_{2}$.

Let $Q: C(J) \rightarrow C_{0}(J)$ and $F: C(J) \rightarrow B_{0}$ be the operators defined by

$$
\begin{gathered}
Q x=x(t)-x\left(t_{0}\right)-\int_{t_{0}}^{t} f(s, x(s)) d s, \\
F x=(Q x, g[x]) .
\end{gathered}
$$

Clearly, $Q x=0$ if and only if $x \in C(J)$ is a solution of (1.1). Thus, the boundary value problem (1.1), (1.2) is equivalent to finding a solution $x \in C(J)$ of the equation

$$
F x=0 \text {. }
$$

In (1.1), (1.2) the function $f(t, x)$ is assumed to be continuous in $J \times R^{n}$ and continuously differentiable with respect to $x$, and $f_{x}(t, x)$ represents the Jacobian matrix of $f(t, x)$ with respect to $x ; g[x]$ is continuously Frèchet differentiable in $C(J)$, and $g_{x}[x]$ denotes the Frèchet derivative of $g$ at $x$.

For $h \in C(J)$, we define the linear operator $F_{x}(x): C(J) \rightarrow B_{0}$ by

$$
F_{x}(x) h=\left(Q_{x}(x) h, g_{x}[x] h\right),
$$

where $F_{x}(x)$ denotes the Frèchet derivative of $F$ at $x$ and

$$
Q_{x}(x) h=h(t)-h\left(t_{0}\right)-\int_{t_{0}}^{t} f_{x}(s, x(s)) h(s) d s .
$$


Let $L \in L\left(C(J), B_{0}\right)$ be the operator independent of $x$ which approximates $F_{x}(x)$ and is defined by

$$
L h=(P h, \ell[h]), \quad h \in C(J)
$$

where the linear operator $P: C(J) \rightarrow C_{0}(J)$ in relation to $Q_{x}(x)$ is

$$
P h=h(t)-h\left(t_{0}\right)-\int_{t_{0}}^{t} A(s) h(s) d s, A \in M[J], \text { and } \ell \in L\left(C(J), R^{n}\right) .
$$

Let $B_{1}$ and $B_{2}$ be two Banach spaces. A linear operator $T: B_{2} \rightarrow B_{1}$ is said to be invertible if the equation $T b_{2}=b_{1}$ has a unique solution $b_{2} \in B_{2}$ for each $b_{1} \in B_{1}$.

LEMMA 2.2. Let $L: B_{1} \rightarrow B_{2}$ be a linear operator and $T: B_{2} \rightarrow B_{1}$ be an invertible linear operator. Then, $L$ is invertible if and only if there exists a nonnegative $n \times n$ matrix $M$, with $\rho(M)<1$, such that

$$
\|I-T L\| \leq M
$$

If $L^{-1}$ exists, then

$$
L^{-1}=\sum_{n=0}^{\infty}(I-T L)^{n} T
$$

and

$$
\left\|L^{-1}\right\| \leq(I-M)^{-1}\|T\| .
$$

Proof. Assume that $T, T^{-1}$ exist and (2.8) is satisfied. Since

$$
\begin{aligned}
\left\|\sum_{n=0}^{\infty}(I-T L)^{n} T\right\| & \leq\|T\|+\|I-T L\|\|T\|+\|I-T L\|^{2}\|T\|+\cdots \\
& \leq\left(I+M+M^{2}+\cdots\right)\|T\|=(I-M)^{-1}\|T\|,
\end{aligned}
$$

the infinite series in (2.9) defines a bounded linear operator in $B_{2}$. Hence, for each $b_{2} \in B_{2}$,

$$
b^{*}=\sum_{n=0}^{\infty}(I-T L)^{n} T b_{2}
$$

is a uniquely defined element of $B_{1}$.

From (2.12), we have

$$
(I-T L) b^{*}=\sum_{n=1}^{\infty}(I-T L)^{n} T b_{2}=b^{*}-T b_{2},
$$

and thus

$$
T L b^{*}=T b_{2} \text {. }
$$


Since $T^{-1}$ exists, $L b^{*}=b_{2}$, so that $L b_{1}=b_{2}$ has at least one solution $b_{1}=b^{*}$ for each $b_{2} \in B_{2}$. To show that this solution is unique, we assume that $L b_{1}=b_{2}$ and $b_{1} \neq b^{*}$. Then, $L\left(b_{1}-b^{*}\right)=0$ and hence

$$
(I-T L)\left(b_{1}-b^{*}\right)=b_{1}-b^{*} \neq 0,
$$

which implies that $\rho(\|I-T L\|) \geq 1$. But since $\rho(M)<1$, this contradicts the assumption (2.8). Hence, $L^{-1}$ exists and is given by (2.9). Inequality (2.10) follows immediately from (2.11) and (2.9).

To prove the necessity part, we assume that $L^{-1}$ exists. For $T=L^{-1}$ it is clear that $T^{-1}=L$ exists and

$$
\|I-T L\|=\|I-I\|=0 \leq M,
$$

so that $(2.8)$ is satisfied.

COROLLARY 2.3. In Lemma 2.2, (2.8)-(2.10) can be replaced by

$$
\begin{gathered}
\|I-T L\| \leq M, \\
L^{-1}=\sum_{n=0}^{\infty} T(I-L T)^{n}
\end{gathered}
$$

and

$$
\left\|L^{-1}\right\| \leq\|T\|(I-M)^{-1}
$$

respectively.

\section{Linear problems}

Let $A \in M[J]$ and $\Phi(t)$ be the fundamental matrix solution of the homogeneous system $y^{\prime}=A(t) y$ such that $\Phi\left(t_{0}\right)=I$. For $\ell \in L\left(C(J), R^{n}\right)$ we define an $n \times n$ matrix $G$ by

$$
G=\ell[\Phi],
$$

whose column vectors are $\ell \phi_{i} ; i=1, \ldots, n$ and $\phi_{i}$ is the $i$-th column vector of $\Phi$. If $G$ is nonsingular, then we shall denote by

$$
S=\Phi G^{-1} .
$$

For $h \in C(J)$, let $E$ be the element of $L(C(J), C(J))$ defined by

$$
E h=\int_{t_{0}}^{t} \Phi(t) \Phi^{-1}(s) h(s) d s .
$$


Let $K: C(J) \rightarrow C(J)$ be the operator defined by

$$
K x=f(t, x(t))-A(t) x(t), \quad x \in C(J) .
$$

Now for $h \in C(J), y=(u, e) \in B_{0}$, we consider the system

$$
P h=u(t),
$$

together with

$$
\ell[h]=e .
$$

LEMMA 3.1. If the matrix $G$ is nonsingular, then (3.5), (3.6) has a unique solution $h(t)$, that is, for the operator $L$ defined in (2.6), $L^{-1}$ exists, and can be represented as

$$
L^{-1} y=h(t)=S_{1} E_{1} u+S e,
$$

where

$$
E_{1}=I+E A, \quad S_{1}=I-S \ell, \quad H=S_{1} E .
$$

PROOF. Any solution of (3.5) can be expressed as

$$
h(t)=\Phi(t) c+u(t)+\Phi(t) \int_{t_{0}}^{t} \Phi^{-1}(s) A(s) u(s) d s,
$$

where $c$ is an arbitrary constant vector. The solution (3.9) satisfies (3.6) if and only if

$$
G c+\ell(I+E A) u=e .
$$

Since $\operatorname{det} G \neq 0$, from (3.10) we get

$$
c=G^{-1} e-G^{-1} \ell(I+E A) u .
$$

Substituting this in (3.9) and following the definitions of $S, E_{1}$ and $S_{1}$, the result (3.7) follows.

COROLLARY 3.2. Assume that the matrix $G$ is nonsingular, and for $\phi \in C(J)$, let

$$
T \phi=\phi-S_{1} E_{1}[Q \phi]-S[g[\phi]] .
$$

Then

$$
T \phi=(H K+S(\ell-g)) \phi .
$$

ProOF. Since an integration by parts of $S_{1} E_{1}=[I+H A-S \ell]$ gives that $S_{1} E_{1} Q \phi=$ $[I-H K-S \ell] \phi$, it follows that

$$
T \phi=\phi-[I-H K-S \ell] \phi-S[g[\phi]]=[H K+S(\ell-g)] \phi .
$$


In the existence and uniqueness theory of solutions of (1.1), (1.2) matrices $\Phi(t)$ and $\Phi^{-1}(t)$ play a vital role. However, in practical evaluation it often becomes necessary to approximate these matrices by the computed fundamental matrices. Let $\tilde{\Phi}(t)$ and $\hat{\Phi}(t)$ be the matrices that approximate $\Phi(t)$ and $\Phi^{-1}(t)$, respectively. Hereafter, for an operator $Z=Z\left(\Phi, \Phi^{-1}\right)$ depending on $\Phi$ and $\Phi^{-1}$, we shall denote by $\tilde{Z}$ the operator $Z(\tilde{\Phi}, \hat{\Phi})$.

We shall consider the following two cases:

Case 1. $\tilde{\Phi}(t)$ and $\hat{\Phi}(t)$ are continuous on $J$.

Case 2. $\tilde{\Phi}(t)$ and $\hat{\Phi}(t)$ are continuously differentiable on $J$.

Case 1. Let

$$
\begin{gathered}
\Gamma(t)=\tilde{\Phi}(t) \hat{\Phi}(t), \\
\Gamma_{1}(t)=I-\Gamma(t), \\
\sigma=\max \left(b-t_{0}, t_{0}-a\right), \\
\Psi(t)=\tilde{\Phi}(t)-I-\int_{t_{0}}^{t} A(s) \tilde{\Phi}(s) d s, \\
\Psi_{1}(t)=\hat{\Phi}(t)-I+\int_{t_{0}}^{t} \hat{\Phi}(s) A(s) d s, \\
\Psi_{2}(t)=\Gamma_{1}(t)+\tilde{\Phi}(t) \Psi_{1}(t) .
\end{gathered}
$$

Let $R, R_{1} \in L(C(J), C(J))$ and $R_{2}: C(J) \rightarrow C(J)$ be the operators defined by

$$
\begin{gathered}
R h=\Psi_{2}(t) \int_{t_{0}}^{t} h(s) d s-\tilde{\Phi}(t) \int_{t_{0}}^{t} \Psi_{1}(s) h(s) d s, \quad h \in C(J) \\
R_{1} h=\Psi_{2} h\left(t_{0}\right)+R A h, \quad h \in C(J) \\
R_{2} x=\Psi_{2} x\left(t_{0}\right)+R f(t, x(t)), \quad x \in C(J) .
\end{gathered}
$$

LEMMA 3.3. Assume that

$$
\begin{gathered}
\tilde{G} \text { is nonsingular, } \\
\left\|\tilde{G}^{-1}\right\|\|\ell\| \exp (\sigma\|A\|)\|\Psi\| \leq M,
\end{gathered}
$$

and also

$$
\left\|\tilde{S}_{1} R_{1}\right\| \leq \bar{M},
$$

where $M$ and $\bar{M}$ are nonnegative $n \times n$ matrices with $\rho(M)<1$ and $\rho(\bar{M})<1$. Then $\tilde{L}^{-1}$ exists and is invertible. 
ProOF. Clearly (3.22) implies that $\tilde{L}^{-1}$ is defined. Since

$$
\Phi(t)-I-\int_{t_{0}}^{t} A(s) \Phi(s) d s=0
$$

from (3.16), we have

$$
\tilde{\Phi}(t)-\Phi(t)=\Psi(t)+\int_{t_{0}}^{t} A(s)[\tilde{\Phi}(s)-\Phi(s)] d s
$$

and hence

$$
\|\tilde{\Phi}(t)-\Phi(t)\| \leq\|\Psi\|+\left|\int_{t_{0}}^{t}\|A\|\|\tilde{\Phi}(s)-\Phi(s)\| d s\right| .
$$

Thus, by Gronwall's inequality we find that

$$
\|\tilde{\Phi}-\Phi\| \leq \exp (\sigma\|A\|)\|\Psi\|
$$

and since

$$
\left\|I-\tilde{G}^{-1} G\right\| \leq\left\|\tilde{G}^{-1}\right\|\|\tilde{G}-G\| \leq\left\|\tilde{G}^{-1}\right\|\|\ell\|\|\tilde{\Phi}-\Phi\|,
$$

in view of (3.25), (3.23) and Lemma 2.2, it follows that $G$ is nonsingular, and in conclusion $L$ is invertible.

We will now show that

$$
\left\|I-\tilde{L}^{-1} L\right\| \leq \bar{M}
$$

Let

$$
\Lambda(t)=\hat{\Phi}(t)-\Phi^{-1}(t), \quad \text { and } \quad \Omega(t)=\int_{t_{0}}^{t} \Lambda(s) A(s) d s
$$

Since

$$
\Phi^{-1}(t)-I+\int_{t_{0}}^{t} \Phi^{-1}(s) A(s) d s=0
$$

by (3.17) we have

$$
\Lambda(t)+\Omega(t)=\Psi_{1}(t) .
$$

Let $u(t)=\int_{t_{0}}^{t} p(s) d s, p \in C(J)$. In view of $\left(\Phi^{-1}\right)^{\prime}=-\Phi^{-1} A$, an integration by parts gives that

$$
\int_{t_{0}}^{t} \Phi^{-1}(s) A(s) u(s) d s=-\Phi^{-1}(t) u(t)+\int_{t_{0}}^{t} \Phi^{-1}(s) p(s) d s
$$


and

$$
\int_{t_{0}}^{t} \Lambda(s) A(s) u(s) d s=\Omega(t) u(t)-\int_{t_{0}}^{t} \Omega(s) p(s) d s .
$$

Thus, by (3.27)-(3.30) we have

$$
\begin{aligned}
\tilde{E} A u & =\tilde{\Phi}(t)\left[\int_{t_{0}}^{t} \Phi^{-1}(s) A(s) u(s) d s+\int_{t_{0}}^{t} \Lambda(s) A(s) u(s) d s\right] \\
& =-u+\tilde{E} p+R p .
\end{aligned}
$$

From this and (3.20), we have

$$
\tilde{E}_{1} P h=h-\tilde{\Phi} h\left(t_{0}\right)-R_{1} h, \quad h \in C(J) .
$$

Finally, since by (3.1) and (3.2)

$$
\tilde{S}_{1} \tilde{\Phi}=\left(I-\tilde{\Phi} \tilde{G}^{-1} \ell\right) \tilde{\Phi}=0
$$

from (3.7), (3.32) and (3.33) for $h \in C(J)$, we find that

$$
\left(I-\tilde{L}^{-1} L\right) h=h-\tilde{S}_{1} \tilde{E}_{1} P h-\tilde{S} \ell[h]=\tilde{S}_{1} R_{1} h .
$$

Hence, in view of (3.24) we find that (3.26) holds. Corollary 2.3 now implies that $\tilde{L}^{-1}$ is invertible.

COROLLARY 3.4. Assume that (3.22) holds and let

$$
\tilde{T} \phi=\phi-\tilde{S}_{1} \tilde{E}_{1}[Q \phi]-\tilde{S}[g[\phi]], \quad \phi \in C(J) .
$$

Then

$$
\tilde{T} \phi=\left[\tilde{H} K+\tilde{S}(\ell-g)+\tilde{S}_{1} R_{2}\right] \phi .
$$

ProOF. By (3.31), we have

$$
\tilde{E}_{1} Q \phi=\phi-\tilde{E} K \phi-\tilde{\Phi} \phi\left(t_{0}\right)-R_{2} \phi
$$

and hence (3.33) gives that

$$
\tilde{S}_{1} \tilde{E}_{1} Q \phi=\phi-\tilde{S} \ell[\phi]-\tilde{H} K \phi-\tilde{S}_{1} R_{2} \phi .
$$

Substituting (3.37) into (3.34), we obtain (3.35). 
Case 2. Let

$$
\begin{gathered}
A_{1}(t)=\tilde{\Phi}^{\prime}(t) \tilde{\Phi}^{-1}(t), \\
A_{2}(t)=-\hat{\Phi}^{-1}(t) \hat{\Phi}^{\prime}(t)
\end{gathered}
$$

$P_{2}, R_{3}, R_{4} \in L(C(J), C(J))$ and $R_{5}: C(J) \rightarrow C(J)$ be the operators defined by

$$
\begin{gathered}
P_{2} h=h(t)-h\left(t_{0}\right)-\int_{t_{0}}^{t} A_{1}(s) h(s) d s, \quad h \in C(J), \\
R_{3} h=\tilde{E}\left(A-A_{2}\right) h+\Gamma_{1} h, \quad h \in C(J), \\
R_{4} h=R_{3}\left(h-P_{2} h\right)-\tilde{E}\left(A-A_{1}\right) h, \quad h \in C(J), \\
R_{5} x=R_{3}(x-Q x), \quad x \in C(J) .
\end{gathered}
$$

LEMMA 3.5. Assume that (3.22) holds. Then $\tilde{L}^{-1}$ is invertible if

$$
\sigma\left\|A_{1}-\Gamma A\right\| \leq N
$$

or

$$
\left\|\tilde{S}_{1} R_{4}\right\| \leq \bar{N}
$$

where $N$ and $\bar{N}$ are $n \times n$ nonnegative matrices with $\rho(N)<1$ and $p(\bar{N})<1$.

PROOF. Let $L_{1}$ be the operator defined by

$$
L_{1} h=\left(P_{2} h, \ell[h]\right), \quad h \in C(J) .
$$

Clearly, in view of (3.22), this operator $L_{1}$ is invertible.

For any $y=(u, e) \in B_{0}$ by (3.7), we find that

$$
\tilde{L}^{-1} y=\tilde{E}_{1} u-\tilde{S}\left[\ell\left[\tilde{E}_{1} u\right]-e\right] .
$$

Since $P_{2} \tilde{\Phi}=0$ and $\tilde{G}=\ell[\tilde{\Phi}]$, we have

$$
P_{2} \tilde{S}=\left(P_{2} \tilde{\Phi}\right) \tilde{G}^{-1}=0
$$

and

$$
\ell[\tilde{S}]=\ell[\tilde{\Phi}]) \tilde{G}^{-1}=I .
$$

Now suppose that (3.44) holds. By (3.47) and (3.49), we get

$$
\ell\left[\tilde{L}^{-1} y\right]=e
$$


and by (3.47) and (3.48), $P_{2} \tilde{L}^{-1} y=P_{2} \tilde{E}_{1} u$. Since $\tilde{\Phi}^{\prime}=A_{1} \tilde{\Phi}$ and $u \in C_{0}(J)$, integration by parts gives that

$$
P_{2} \tilde{L}^{-1} y=P_{2} \tilde{E}_{1} u=u(t)-\int_{t_{0}}^{t}\left[A_{1}(s)-\Gamma(s) A(s)\right] u(s) d s .
$$

Using this relation and (3.50), we find that

$$
\left(I-L_{1} \tilde{L}^{-1}\right) y=\left(\int_{t_{0}}^{t}\left[A_{1}(s)-\Gamma(s) A(s)\right] u(s) d s, 0\right) .
$$

Thus, it follows that $\left\|\left(I-L_{1} \tilde{L}^{-1}\right) y\right\| \leq \sigma\left\|A_{1}-\Gamma A\right\|\|y\|$ and hence (3.44) and Lemma 2.2 implies that $\tilde{L}^{-1}$ is invertible.

Next suppose that (3.45) holds. For $q \in C(J)$, let $u(t)=\int_{t_{0}}^{t} q(s) d s$. Since $\hat{\Phi}^{\prime}=-\hat{\Phi} A_{2}$, integration by parts gives that

$$
\tilde{E} A u=\tilde{E}\left(A-A_{2}\right) u-\Gamma u+\tilde{E} q=-u+\tilde{E} q+R_{3} u .
$$

By this, for $h \in C(J)$ we get

$$
\tilde{E}_{1} P_{2} h=h-\tilde{\Phi} \hat{\Phi}\left(t_{0}\right) h\left(t_{0}\right)-R_{4} h .
$$

Now substituting $u=P_{2} h$ and $e=\ell[h]$ into (3.47) and using the resulting relation together with (3.52) and (3.33), we obtain $\left(I-\tilde{L}^{-1} L_{1}\right) h=\tilde{S}_{1} R_{4} h$. Thus, (3.45) and Corollary 2.3 imply that $\tilde{L}^{-1}$ is invertible.

COROLlaRY 3.6. Assume that (3.22) holds and let $\tilde{T} \phi$ be as in (3.34). Then

$$
\tilde{T} \phi=\left[\tilde{H} K+\tilde{S}(\ell-g)+\tilde{S}_{1} R_{5}\right] \phi .
$$

Proof. By (3.51), we have

$$
\tilde{E}_{1} Q \phi=\phi-\tilde{E} K \phi-\tilde{\Phi} \hat{\Phi}\left(t_{0}\right) \phi\left(t_{0}\right)-R_{5} \phi
$$

and hence (3.33) gives that

$$
\tilde{S}_{1} \tilde{E}_{1} Q \phi=\phi-\tilde{S} \ell[\phi]-\tilde{H} K \phi-\tilde{S}_{1} R_{5} \phi .
$$

Substituting (3.55) into (3.34), we get (3.53). 


\section{Convergence of Picard's Method}

Using no approximation of Green's function in our earlier paper [20] we proved the following result, its proof being based on Theorem 2.1.

THEOREM 4.1. With respect to the boundary value problem (2.3) assume that there is an approximate solution $\bar{x}(t) \in C(J)$, and

(i) there exists an $n \times n$ continuous matrix $A(t)$ and a bounded continuous linear operator $\ell$ such that $G=\ell[\Phi(t)]$ is nonsingular, where $\Phi(t)$ is the fundamental matrix solution of the homogeneous differential system $y^{\prime}=$ $A(t) y$,

(ii) there exist nonnegative $n \times n$ matrices $M_{1}$ and $M_{2}$ such that $\|H\| \leq M_{1}$ and $\|S\| \leq M_{2}$,

(iii) there exist nonnegative $n \times n$ matrices $M_{3}$ and $M_{4}$ and a positive vector $r$ such that for all $x(t) \in \bar{S}(\bar{x}, r)=\{z \in C(J):\|z-\bar{x}\| \leq r\},\left\|f_{x}(t, x(t))-A(t)\right\|$ $\leq M_{3}$ and $\left\|g_{x}[x]-\ell\right\| \leq M_{4}$,

(iv) there exists a nonnegative vector $\eta_{0}$ such that $\left\|S_{1} E_{1}[Q \bar{x}]+S[g[\bar{x}]]\right\| \leq \eta_{0}$,

(v) $K_{0}=M_{1} M_{3}+M_{2} M_{4}, \rho\left(K_{0}\right)<1$ and $r_{0}=\left(I-K_{0}\right)^{-1} \eta_{0} \leq r$.

Then

(1) there exists a solution $x^{*}(t)$ of $(2.3)$ in $\bar{S}\left(\bar{x}, r_{0}\right)$,

(2) $x^{*}(t)$ is the unique solution of $(2.3)$ in $\bar{S}(\bar{x}, r)$,

(3) the sequence $\left\{x_{m}(t)\right\}$ defined by

$$
\begin{aligned}
& x_{m+1}(t)=x_{m}(t)-S_{1} E_{1}\left[Q x_{m}\right]-S\left[g\left[x_{m}\right]\right], \\
& x_{0}(t)=\bar{x}(t) ; \quad m=0,1, \ldots .
\end{aligned}
$$

converges to $x^{*}(t)$ with

$$
\left\|x^{*}-x_{m}\right\| \leq K_{0}^{m} r_{0}
$$

(4) for $x_{0}(t)=x(t) \in \bar{S}\left(\bar{x}, r_{0}\right)$ the iterative process (4.1) converges to $x^{*}(t)$,

(5) any sequence $\left\{\bar{x}_{m}(t)\right\}$ such that $\bar{x}_{m}(t) \in \bar{S}\left(x_{m}, K_{0}^{m} r_{0}\right) ; m=0,1, \ldots$ converges to $x^{*}(t)$.

Now we shall discuss the convergence of Picard's method when the fundamental matrices are replaced by the approximate fundamental matrices. First we shall consider Case 1.

THEOREM 4.2. With respect to the boundary value problem (2.3) we assume that there is an approximate solution $\bar{x}(t) \in C(J)$ and

(i) $\tilde{L}^{-1}$ is invertible, 
(ii) there exist nonnegative $n \times n$ matrices $M_{1}$ and $M_{2}$ such that $\|\tilde{H}\| \leq M_{1}$ and $\|\tilde{S}\| \leq M_{2}$,

(iii) there exist nonnegative $n \times n$ matrices $M_{3}, M_{4}$ and $M_{5}$ and a positive vector $r$ such that for all $x(t) \in \bar{S}(\bar{x}, r)=\{z \in C(J):\|z-\bar{x}\| \leq r\}, \| f_{x}(t, x(t))$ $-A(t)\left\|\leq M_{3},\right\| g_{x}[x]-\ell \| \leq M_{4}$ and $\left\|f_{x}(t, x(t))\right\| \leq M_{5}$,

(iv) there exist nonnegative $n \times n$ matrices $M_{6}, M_{7}$ such that $\left\|\tilde{S}_{1} R\right\| \leq M_{6}$ and $\left\|\tilde{S}_{1} \Psi_{2}\right\| \leq M_{7}$,

(v) there exists a nonnegative vector $\eta_{1}$ such that

$$
\left\|\tilde{S}_{1} \tilde{E}_{1}[Q \bar{x}]+\tilde{S}[g[\bar{x}]]\right\| \leq \eta_{1},
$$

(vi) $K_{1}=M_{1} M_{3}+M_{2} M_{4}+M_{6} M_{5}+M_{7}, \rho\left(K_{1}\right)<1$ and $r_{1}=\left(I-K_{1}\right)^{-1} \eta_{1} \leq r$.

Then

(1) there exists a solution $x^{*}(t)$ of $(2.3)$ in $\bar{S}\left(\bar{x}, r_{1}\right)$,

(2) $x^{*}(t)$ is the unique solution of $(2.3)$ in $\bar{S}(\bar{x}, r)$,

(3) the sequence $\left\{u_{m}(t)\right\}$ defined by

$$
\begin{gathered}
u_{m+1}(t)=\tilde{H}\left[K u_{m}\right]+\tilde{S}\left[\ell\left[u_{m}\right]-g\left[u_{m}\right]\right]+\tilde{S}_{1}\left[R f\left(t, u_{m}(t)\right)+\Psi_{2} u_{m}\left(t_{0}\right)\right], \\
u_{0}(t)=x_{0}(t)=\bar{x}(t), \quad m=0,1, \ldots,
\end{gathered}
$$

converges to $x^{*}(t)$ with $\left\|x^{*}-u_{m}\right\| \leq K_{1}^{m} r_{1}$,

(4) for $u_{0}(t)=x(t) \in \bar{S}\left(\bar{x}, r_{1}\right)$ the iterative process (4.2) converges to $x^{*}(t)$,

(5) any sequence $\left\{\bar{u}_{m}(t)\right\}$ such that $\bar{u}_{m}(t) \in \bar{S}\left(u_{m}, K_{1}^{m} r_{1}\right), m=0,1, \ldots$, converges to $x^{*}(t)$.

ProOF. Define an operator $\tilde{T}: \bar{S}(\bar{x}, r) \rightarrow C(J)$ by

$$
\tilde{T} x(t)=\tilde{H}[K x]+\tilde{S}[\ell[x]-g[x]]+\tilde{S}_{1}\left[R f(t, x(t))+\Psi_{2} x\left(t_{0}\right)\right] .
$$

If $x(t)$ is a solution of (4.3), that is, $\tilde{T} x(t)=x(t)$, then from Corollary 3.4 it is clear that $\tilde{S}_{1} \tilde{E}_{1}[Q x]+\tilde{S}[g[x]]=0$. But this is the same as $\tilde{L}^{-1} F x=0$. Thus, in view of condition (i) it follows that $F x=0$, that is, $x(t)$ is a solution of (2.3). Thus, it suffices to show that the operator $\tilde{T}$ satisfies the conditions of Theorem 2.1. For this, if $x(t), y(t) \in \bar{S}(\bar{x}, r)$ then we have

$$
\begin{aligned}
\tilde{T} x(t)-\tilde{T} y(t)=\tilde{H}\left[\int_{0}^{1}\left[f_{x}\left(t, x(t)+\theta_{1}(y(t)-x(t))\right)-A(t)\right](x(t)-y(t)) d \theta_{1}\right] \\
+\tilde{S}\left[\int_{0}^{1}\left[\ell-g_{x}\left(x+\theta_{2}(y-x)\right)\right][x-y] d \theta_{2}\right] \\
+\tilde{S}_{1}\left[R \int_{0}^{1} f_{x}\left(t, x(t)+\theta_{1}(y(t)-x(t))\right)(x(t)-y(t)) d \theta_{1}\right. \\
\left.+\Psi_{2}\left(x\left(t_{0}\right)-y\left(t_{0}\right)\right)\right] .
\end{aligned}
$$


Therefore, it follows that

$$
\|\tilde{T} x-\tilde{T} y\| \leq\left(M_{1} M_{3}+M_{2} M_{4}+M_{6} M_{5}+M_{7}\right)\|x-y\|=K_{1}\|x-y\| .
$$

Next from Corollary 3.4 and (4.3), we have

$$
\tilde{T} x_{0}(t)-x_{0}(t)=\tilde{T} \bar{x}(t)-\bar{x}(t)=-\tilde{S}_{1} \tilde{E}_{1}[Q \bar{x}]-\tilde{S}[g[\bar{x}]]
$$

and hence

$$
\left\|\tilde{T} x_{0}-x_{0}\right\| \leq\left\|-\tilde{S}_{1} \tilde{E}_{1}[Q \bar{x}]-\tilde{S}[g[\bar{x}]]\right\| \leq \eta_{1} .
$$

Thus we find that $\left(I-K_{1}\right)^{-1}\left\|\tilde{T} x_{0}-x_{0}\right\| \leq\left(I-K_{1}\right)^{-1} \eta_{1}=r_{1} \leq r$. Hence the conditions of Theorem 2.1 are satisfied and the conclusions (1)-(5) follow.

REMARK 4.1. For computational purposes let us assume the following: $P_{1}$ and $P_{2}$ are $n \times n$ nonnegative matrices such that

$$
\begin{gathered}
\sigma\left(\left\|\Psi_{2}\right\|\|A\|+\|\tilde{\Phi}\|\left\|\Psi_{1} A\right\|\right) \leq P_{1}, \\
\sigma\left(\left\|\Psi_{2}\right\|+\|\tilde{\Phi}\|\left\|\Psi_{1}\right\|\right) \leq P_{2} .
\end{gathered}
$$

Then, for any $h \in C(J)$ by (3.19), $\|R A h\| \leq P_{1}\|h\|$, and $\|R h\| \leq P_{2}\|h\|$. Hence (3.24) can be replaced by

$$
\left\|\tilde{S}_{1} \Psi_{2}\right\|+\left\|\tilde{S}_{1}\right\| P_{1} \leq \bar{M}
$$

Also, $\left\|\tilde{S}_{1} R\right\| \leq M_{6}$ in (iv) of Theorem 4.2 can be changed to

$$
\left\|\tilde{S}_{1}\right\| P_{2} \leq M_{6}
$$

Next we shall consider Case 2.

THEOREM 4.3. With respect to the boundary value problem (2.3) we assume that there is an approximate solution $\bar{x}(t) \in C(J)$, and

(i) the conditions (i)-(iii) and (v) of Theorem 4.2 are satisfied,

(ii) there exists an $n \times n$ nonnegative matrix $M_{8}$ such that $\left\|\tilde{S}_{1} R_{3}\right\| \leq M_{8}$,

(iii) $K_{2}=M_{1} M_{3}+M_{2} M_{4}+M_{8}\left(I+\sigma M_{5}\right) ; \rho\left(K_{2}\right)<1$ and $r_{2}=\left(I-K_{2}\right)^{-1} \eta_{1} \leq r$.

Then

(1) there exists a solution $x^{*}(t)$ of $(2.3)$ in $\bar{S}\left(\bar{x}, r_{2}\right)$,

(2) $x^{*}(t)$ is the unique solution of (2.3) in $\bar{S}(\bar{x}, r)$, 
(3) the sequence $\left\{u_{m}(t)\right\}$ defined by

$$
\begin{array}{r}
u_{m+1}(t)=\tilde{H}\left[K u_{m}\right]+\tilde{S}\left[\ell\left[u_{m}\right]-g\left[u_{m}\right]\right]+\tilde{S}_{1}\left[R_{3}\left(u_{m}-Q u_{m}\right)\right], \\
u_{0}(t)=x_{0}(t)=\bar{x}(t), \quad m=0,1, \ldots,
\end{array}
$$

converges to $x^{*}(t)$ with $\left\|x^{*}-u_{m}\right\| \leq K_{2}^{m} r_{2}$,

(4) for $u_{0}(t)=x(t) \in \bar{S}\left(\bar{x}, r_{2}\right)$ the iterative process (4.6) converges to $x^{*}(t)$,

(5) any sequence $\left\{\bar{u}_{m}(t)\right\}$ such that $\bar{u}_{m}(t) \in \bar{S}\left(u_{m}, K_{2}^{m} r_{2}\right), m=0,1, \ldots$, converges to $x^{*}(t)$.

PROOF. Define an operator $\tilde{T}: \bar{S}(\bar{x}, r) \rightarrow C(J)$ by

$$
\tilde{T} x(t)=\tilde{H}[K x]+\tilde{S}[\ell[x]-g[x]]+\tilde{S}_{1}\left[R_{3}(x-Q x)\right] .
$$

Now as in Theorem 4.2, we can show that the operator $\tilde{T}$ satisfies the conditions of Theorem 2.1.

REMARK 4.2. Again for computational purposes we assume that $\rho\left(\left\|\Gamma_{1}\right\|\right)<1$ and let $Z=\left(I-\left\|\Gamma_{1}\right\|\right)^{-1}$. Then, since from Lemma $2.2,\left\|\Gamma^{-1}\right\| \leq Z$ we have the following:

$$
\begin{aligned}
\left\|A_{1}-\Gamma A\right\| & \leq\left\|\tilde{\Phi}^{\prime} \hat{\Phi}-\Gamma A \Gamma\right\| Z, \\
\left\|A-A_{1}\right\| & \leq\left\|A \Gamma-\tilde{\Phi}^{\prime} \hat{\Phi}\right\| Z, \\
\left\|A-A_{2}\right\| & \leq Z\left\|\Gamma A+\tilde{\Phi} \hat{\Phi}^{\prime}\right\|, \\
\left\|A_{1}\right\| & \leq\left\|\tilde{\Phi}^{\prime} \hat{\Phi}\right\| Z .
\end{aligned}
$$

Now let $P_{3}$ be an $n \times n$ nonnegative matrix such that

$$
\|\tilde{H}\|\left\|A-A_{2}\right\|+\left\|\tilde{S_{1}}\right\|\left\|\Gamma_{1}\right\| \leq P_{3},
$$

then

$$
\left\|\tilde{S}_{1} R_{3} h\right\| \leq P_{3}\|h\|, \quad h \in C(J)
$$

Hence it follows that

$$
\left\|\tilde{S}_{1} R_{4}\right\| \leq P_{3}\left(I+\sigma\left\|A_{1}\right\|\right)+\|\tilde{H}\|\left\|A-A_{1}\right\|
$$

and

$$
\left\|\tilde{S}_{1} R_{3}\right\|\left(I+\sigma M_{5}\right) \leq P_{3}\left(I+\sigma M_{5}\right)
$$

Thus, by (4.8)-(4.15) we can compute the left sides of (3.44), (3.45) and (ii) of Theorem 4.3 without computing $\tilde{\Phi}^{-1}$ and $\hat{\Phi}^{-1}$.

REMARK 4.3. If $\tilde{\Phi}(t)=\Phi(t)$ and $\hat{\Phi}(t)=\Phi^{-1}(t)$, then both the Theorems 4.2 and 4.3 reduce to Theorem 4.1 . 


\section{Convergence of approximate Picard's Method}

In our earlier paper [20], we have provided necessary and sufficient conditions for the convergence of the approximate Picard's sequence $\left\{y_{m}(t)\right\}$ generated by the scheme

$$
\begin{gathered}
y_{m+1}(t)=y_{m}(t)-S_{1} E_{1}\left[y_{m}(t)-y_{m}\left(t_{0}\right)-\int_{t_{0}}^{t} f_{m}\left(s, y_{m}(s)\right) d s\right]-S\left[g_{m}\left[y_{m}\right]\right], \\
y_{0}(t)=x_{0}(t)=\bar{x}(t), \quad m=0,1, \ldots,
\end{gathered}
$$

to the solution $x^{*}(t)$ of the boundary value problem (2.3). In (5.1) for each $m$, the function $f_{m}$ and the operator $g_{m}$, respectively, approximate $f$ and $g$ and are assumed to be continuous.

In relation to the function $f_{m}$ we define $Q_{m}: C(J) \rightarrow C_{0}(J)$ by

$$
Q_{m} x=x(t)-x\left(t_{0}\right)-\int_{t_{0}}^{t} f_{m}(s, x(s)) d s, \quad x \in C(J), \quad m=0,1, \ldots
$$

LEMMA 5.1. Assume that the matrix $\tilde{G}$ is nonsingular and let

$$
\tilde{T} \phi=\phi-\tilde{S}_{1} \tilde{E}_{1}\left[Q_{m} \phi\right]-\tilde{S}\left[g_{m}[\phi]\right], \quad \phi \in C(J) .
$$

Then for Case 1,

$$
\begin{aligned}
\tilde{T} \phi= & \tilde{H}\left[f_{m}(t, \phi(t))-A(t) \phi(t)\right]+\tilde{S}\left[\ell[\phi]-g_{m}[\phi]\right] \\
& +\tilde{S}_{1}\left[R f_{m}(t, \phi(t))+\Psi_{2} \phi\left(t_{0}\right)\right],
\end{aligned}
$$

and for Case 2,

$$
\tilde{T} \phi=\tilde{H}\left[f_{m}(t, \phi(t))-A(t) \phi(t)\right]+\tilde{S}\left[\ell[\phi]-g_{m}[\phi]\right]+\tilde{S}_{1}\left[R_{3}\left(\phi-Q_{m} \phi\right)\right] .
$$

ProOF. The proof of (5.2) is similar to that of Corollary 3.4, whereas (5.3) follows as in Corollary 3.6.

We shall now consider the following approximate Picard's scheme for Case 1.

$$
\begin{aligned}
v_{m+1}(t)=\tilde{H}\left[f_{m}\left(t, v_{m}(t)\right)-A(t) v_{m}(t)\right]+\tilde{S}\left[\ell\left[v_{m}\right]-g_{m}\left[v_{m}\right]\right] \\
+\tilde{S}_{1}\left[R f_{m}\left(t, v_{m}(t)\right)+\Psi_{2} v_{m}\left(t_{0}\right)\right], \\
v_{0}(t)=x_{0}(t)=\bar{x}(t), \quad m=0,1, \ldots
\end{aligned}
$$

In (5.4) once again for each $m$, the function $f_{m}$ and the operator $g_{m}$ are assumed to be continuous. In addition, with respect to $f_{m}$ and $g_{m}$ we shall assume that the following conditions are satisfied. 
CONDITION $c_{1}$ : For all $t \in J$ and each $v_{m}(t)$ obtained from (5.4) the inequality

$$
\left|f\left(t, v_{m}(t)\right)-f_{m}\left(t, v_{m}(t)\right)\right| \leq \Delta_{1}\left|f\left(t, v_{m}(t)\right)\right|
$$

holds, where $\Delta_{1}$ is an $n \times n$ nonnegative matrix with $\rho\left(\Delta_{1}\right)<1$.

CONDITION $c_{2}$ : For each $v_{m}(t)$ obtained from (5.4) the inequality

$$
\left\|g\left[v_{m}\right]-g_{m}\left[v_{m}\right]\right\| \leq \Delta_{2}\left\|g\left[v_{m}\right]\right\|
$$

holds, where $\Delta_{2}$ is an $n \times n$ nonnegative matrix with $\rho\left(\Delta_{2}\right)<1$.

Inequalities (5.5) and (5.6) correspond to the relative error in approximating $f$ and $g$ by $f_{m}$ and $g_{m}$. Further, the above inequalities respectively imply that

$$
\left|f\left(t, v_{m}(t)\right)\right| \leq\left(I-\Delta_{1}\right)^{-1}\left|f_{m}\left(t, v_{m}(t)\right)\right|
$$

and

$$
\left\|g\left[v_{m}\right]\right\| \leq\left(I-\Delta_{2}\right)^{-1}\left\|g_{m}\left[v_{m}\right]\right\| .
$$

THEOREM 5.2. With respect to the boundary value problem (2.3) we assume that there exists an approximate solution $\bar{x}(t) \in C(J)$, and

(i) the conditions (i)-(iv) of Theorem (4.2) are satisfied,

(ii) conditions $c_{1}$ and $c_{2}$ are satisfied,

(iii) there exists a nonnegative vector $\bar{\eta}_{1}$ such that $\left\|\tilde{S}_{1} \tilde{E}_{1}\left[Q_{0} \bar{x}\right]+\tilde{S} g_{0}[\bar{x}]\right\| \leq \bar{\eta}_{1}$ and $\eta=\max \left(\eta_{1}, \bar{\eta}_{1}\right)$,

(iv) $\bar{K}_{1}=K_{1}+\left(M_{1}+M_{6}\right) \Delta_{1}\left(M_{3}+\|A(t)\|\right)+M_{2} \Delta_{2}\left(M_{4}+\|\ell\|\right) ; \rho\left(\bar{K}_{1}\right)<1$ and $\bar{r}_{1}=\left(I-\bar{K}_{1}\right)^{-1}\left[\eta+2\left(M_{1}+M_{6}\right) \Delta_{1}\left(I-\Delta_{1}\right)^{-1}\left|f_{0}\left(t, v_{0}(t)\right)\right|+2 M_{2} \Delta_{2}\left(I-\Delta_{2}\right)^{-1}\right.$ $\left.\left\|g_{0}\left[v_{0}\right]\right\|\right] \leq r$.

Then

(1) all the conclusions (1)-(5) of Theorem 4.2 hold,

(2) the sequence $\left\{v_{m}(t)\right\}$ obtained from (5.4) remains in $\bar{S}\left(\bar{x}, \bar{r}_{1}\right)$,

(3) the sequence $\left\{v_{m}(t)\right\}$ converges to $x^{*}(t)$, the solution of (2.3) if and only if $\lim _{m \rightarrow \infty} b_{m}=0$, where

$$
\begin{aligned}
b_{m}=\| v_{m+1}(t) & -\tilde{H}\left[f\left(t, v_{m}(t)\right)-A(t) v_{m}(t)\right]-\tilde{S}\left[\ell\left[v_{m}\right]-g\left[v_{m}\right]\right] \\
& -\tilde{S}_{1}\left[R f\left(t, v_{m}(t)\right)+\Psi_{2} v_{m}\left(t_{0}\right)\right] \|,
\end{aligned}
$$

(4) the following error estimate holds

$$
\begin{aligned}
\left\|v_{m+1}-x^{*}\right\| \leq\left(I-K_{1}\right)^{-1}[ & K_{1}\left\|v_{m+1}-v_{m}\right\| \\
& +\left(M_{1}+M_{6}\right) \Delta_{1}\left(I-\Delta_{1}\right)^{-1}\left|f_{m}\left(t, v_{m}(t)\right)\right| \\
& \left.+M_{2} \Delta_{2}\left(I-\Delta_{2}\right)^{-1}\left\|g_{m}\left[v_{m}\right]\right\|\right] .
\end{aligned}
$$


PROOF. Since $K_{1} \leq \bar{K}_{1}, \rho\left(\bar{K}_{1}\right)<1$ implies that $\rho\left(K_{1}\right)<1$ and $\bar{r}_{1} \geq r_{1}$. Hence the conditions of Theorem 4.2 are satisfied and (1) follows.

To show (2), it is obvious that $\bar{x}(t) \in \bar{S}\left(\bar{x}, \bar{r}_{1}\right)$. Further, from (5.4) and Lemma 5.1 we have

$$
v_{1}(t)-v_{0}(t)=v_{1}(t)-\bar{x}(t)=-\tilde{S}_{1} \tilde{E}_{1}\left[Q_{0} \bar{x}\right]-\tilde{S}\left[g_{0}[\bar{x}]\right],
$$

and hence in view of (iii) and (iv) it follows that $\left\|v_{1}-v_{0}\right\| \leq \bar{\eta}_{1} \leq \eta \leq \bar{r}_{1}$, that is, $v_{1}(t) \in \bar{S}\left(\bar{x}, \bar{r}_{1}\right)$.

Now we shall show that $v_{m+1}(t) \in \bar{S}\left(\bar{x}, \bar{r}_{1}\right)$ provided $v_{m}(t) \in \bar{S}\left(\bar{x}, \bar{r}_{1}\right)$. From (5.4) we have

$$
\begin{aligned}
& v_{m+1}(t)-v_{0}(t)=\left(v_{1}(t)-v_{0}(t)\right) \\
& +\tilde{H}\left[\left(f_{m}\left(t, v_{m}(t)\right)-f\left(t, v_{m}(t)\right)\right)+\left(f\left(t, v_{0}(t)\right)-f_{0}\left(t, v_{0}(t)\right)\right)\right. \\
& \left.\quad+\int_{0}^{1}\left(f_{x}\left(t, v_{0}(t)+\theta_{1}\left(v_{m}(t)-v_{0}(t)\right)\right)-A(t)\right)\left(v_{m}(t)-v_{0}(t)\right) d \theta_{1}\right] \\
& +\tilde{S}\left[\left(g\left[v_{m}\right]-g_{m}\left[v_{m}\right]\right)+\left(g_{0}\left[v_{0}\right]-g\left[v_{0}\right]\right)\right. \\
& \left.\quad-\int_{0}^{1}\left(g_{x}\left[v_{0}+\theta_{2}\left(v_{m}-v_{0}\right)\right]-\ell\right)\left[v_{m}-v_{0}\right] d \theta_{2}\right] \\
& +\tilde{S}_{1}\left[R \left\{\left(f_{m}\left(t, v_{m}(t)\right)-f\left(t, v_{m}(t)\right)\right)+\left(f\left(t, v_{0}(t)\right)-f_{0}\left(t, v_{0}(t)\right)\right)\right.\right. \\
& \left.\left.\quad+\int_{0}^{1} f_{x}\left(t, v_{0}(t)+\theta_{1}\left(v_{m}(t)-v_{0}(t)\right)\right)\left(v_{m}(t)-v_{0}(t)\right) d \theta_{1}\right\}\right] \\
& +\tilde{S}_{1}\left[\Psi_{2}\left(v_{m}\left(t_{0}\right)-v_{0}\left(t_{0}\right)\right)\right] .
\end{aligned}
$$

Thus in view of $v_{0}(t)+\theta_{i}\left(v_{m}(t)-v_{0}(t)\right) \in \bar{S}\left(\bar{x}, \bar{r}_{1}\right), i=1,2$, we find that

$$
\begin{aligned}
\left\|v_{m+1}-v_{0}\right\| \leq & \left\|v_{1}-v_{0}\right\| \\
& +\left(M_{1}+M_{6}\right)\left[\Delta_{1}\left(\left|f\left(t, v_{m}(t)\right)\right|+\left|f\left(t, v_{0}(t)\right)\right|\right)\right] \\
& +M_{2}\left[\Delta_{2}\left(\left\|g\left[v_{m}\right]\right\|+\left\|g\left[v_{0}\right]\right\|\right)\right] \\
& +\left[M_{1} M_{3}+M_{2} M_{4}+M_{6} M_{5}+M_{7}\right]\left\|v_{m}-v_{0}\right\| .
\end{aligned}
$$

However, since

$$
\begin{aligned}
\left|f\left(t, v_{m}(t)\right)\right| \leq & \left|f\left(t, v_{m}(t)\right)-f\left(t, v_{0}(t)\right)-A(t)\left(v_{m}(t)-v_{0}(t)\right)\right| \\
& +\left|f\left(t, v_{0}(t)\right)\right|+\|A(t)\|\left\|v_{m}-v_{0}\right\| \\
\leq & M_{3}\left\|v_{m}-v_{0}\right\|+\|A(t)\|\left\|v_{m}-v_{0}\right\|+\left|f\left(t, v_{0}(t)\right)\right|
\end{aligned}
$$

and similarly

$$
\left\|g\left[v_{m}\right]\right\| \leq M_{4}\left\|v_{m}-v_{0}\right\|+\|\ell\|\left\|v_{m}-v_{0}\right\|+\left\|g\left[v_{0}\right]\right\|,
$$


we obtain

$$
\begin{aligned}
\left\|v_{m+1}-v_{0}\right\| \leq & \bar{\eta}_{1}+\left(M_{1}+M_{6}\right)\left[\Delta_{1}\left(M_{3}+\|A(t)\|\right)\left\|v_{m}-v_{0}\right\|\right. \\
& \left.\quad+2 \Delta_{1}\left(I-\Delta_{1}\right)^{-1}\left|f_{0}\left(t, v_{0}(t)\right)\right|\right] \\
& +M_{2}\left[\Delta_{2}\left(M_{4}+\|\ell\|\right)\left\|v_{m}-v_{0}\right\|+2 \Delta_{2}\left(I-\Delta_{2}\right)^{-1}\left\|g_{0}\left[v_{0}\right]\right\|\right] \\
& +K_{1}\left\|v_{m}-v_{0}\right\| \\
\leq & \bar{K}_{1} \bar{r}_{1}+\left(I-\bar{K}_{1}\right) \bar{r}_{1}=\bar{r}_{1} .
\end{aligned}
$$

Thus, $v_{m+1}(t) \in \bar{S}\left(\bar{x}, \bar{r}_{1}\right)$.

To prove (3), from (4.2) and (5.4) we have

$$
\begin{aligned}
u_{m+1}(t)-v_{m+1}(t)= & -v_{m+1}(t)+\tilde{H}\left[f\left(t, v_{m}(t)\right)-A(t) v_{m}(t)\right] \\
& +\tilde{S}\left[\ell\left[v_{m}\right]-g\left[v_{m}\right]\right]+\tilde{S}_{1}\left[R f\left(t, v_{m}(t)\right)+\Psi_{2} v_{m}\left(t_{0}\right)\right] \\
& +\tilde{H}\left[\int_{0}^{1}\left(f_{x}\left(t, v_{m}(t)+\theta_{1}\left(u_{m}(t)-v_{m}(t)\right)\right)-A(t)\right)\right. \\
& \left.\times\left(u_{m}(t)-v_{m}(t)\right) d \theta_{1}\right] \\
& -\tilde{S}\left[\int_{0}^{1}\left(g_{x}\left[v_{m}+\theta_{2}\left(u_{m}-v_{m}\right)\right]-\ell\right)\left[u_{m}-v_{m}\right] d \theta_{2}\right] \\
+ & {\left[\tilde { S } _ { 1 } \left[R \int_{0}^{1} f_{x}\left(t, v_{m}(t)+\theta_{3}\left(u_{m}(t)-v_{m}(t)\right)\right)\left(u_{m}(t)-v_{m}(t)\right) d \theta_{3}\right.\right.} \\
& \left.+\Psi_{2}\left(u_{m}\left(t_{0}\right)-v_{m}\left(t_{0}\right)\right)\right]
\end{aligned}
$$

and hence

$$
\left\|u_{m+1}-v_{m+1}\right\| \leq b_{m}+K_{1}\left\|u_{m}-v_{m}\right\| .
$$

The above inequality, on using the fact that $\left\|u_{0}-v_{0}\right\|=0$, gives that

$$
\left\|u_{m+1}-v_{m+1}\right\| \leq \sum_{i=0}^{m} K_{1}^{m-i} b_{i} .
$$

Thus, in view of the triangle inequality, we get

$$
\left\|x^{*}-v_{m+1}\right\| \leq \sum_{i=0}^{m} K_{1}^{m-i} b_{i}+\left\|x^{*}-u_{m+1}\right\| .
$$

In (5.11), Theorem 4.2 ensures that $\lim _{m \rightarrow \infty}\left\|x^{*}-u_{m+1}\right\|=0$. Thus the condition $\lim _{m \rightarrow \infty} b_{m}=0$ is necessary and sufficient for the convergence of the sequence $\left\{v_{m}(t)\right\}$ to $x^{*}(t)$ follows from the Toeplitz lemma. 
Finally, we shall prove (4). For this, we have

$$
\begin{aligned}
v_{m+1}(t)-x^{*}(t) & \\
=\tilde{H} & {\left[\left(f_{m}\left(t, v_{m}(t)\right)-f\left(t, v_{m}(t)\right)\right)\right.} \\
& \left.\quad+\int_{0}^{1}\left(f_{x}\left(t, x^{*}(t)+\theta_{1}\left(v_{m}(t)-x^{*}(t)\right)\right)-A(t)\right)\left(v_{m}(t)-x^{*}(t)\right) d \theta_{1}\right] \\
+ & \tilde{S}\left[\left(g\left[v_{m}\right]-g_{m}\left[v_{m}\right]\right)-\int_{0}^{1}\left(g_{x}\left[x^{*}+\theta_{2}\left(v_{m}-x^{*}\right)\right]-\ell\right)\left[v_{m}-x^{*}\right] d \theta_{2}\right] \\
+ & \tilde{S}_{1}\left[R \left[\int_{0}^{1} f_{x}\left(t, x^{*}(t)+\theta_{3}\left(v_{m}(t)-x^{*}(t)\right)\right)\left(v_{m}(t)-x^{*}(t)\right) d \theta_{3}\right.\right. \\
& \left.\left.\quad+\left(f_{m}\left(t, v_{m}(t)\right)-f\left(t, v_{m}(t)\right)\right)\right]\right]+\tilde{S}_{1}\left[\Psi_{2}\left(v_{m}\left(t_{0}\right)-x^{*}\left(t_{0}\right)\right)\right] .
\end{aligned}
$$

Thus it follows that

$$
\begin{aligned}
\left\|v_{m+1}-x^{*}\right\| \leq & M_{1}\left[\Delta_{1}\left|f\left(t, v_{m}(t)\right)\right|+M_{3}\left\|v_{m}-x^{*}\right\|\right] \\
& +M_{2}\left[\Delta_{2}\left\|g\left[v_{m}\right]\right\|+M_{4}\left\|v_{m}-x^{*}\right\|\right] \\
& +M_{6}\left[\Delta_{1}\left|f\left(t, v_{m}(t)\right)\right|+M_{5}\left\|v_{m}-x^{*}\right\|\right]+M_{7}\left\|v_{m}-x^{*}\right\| .
\end{aligned}
$$

Using (5.7), (5.8) and the triangle inequality in (5.12), we obtain

$$
\begin{aligned}
\left\|v_{m+1}-x^{*}\right\| \leq & K_{1}\left\|v_{m+1}-x^{*}\right\|+K_{1}\left\|v_{m+1}-v_{m}\right\| \\
& +\left(M_{1}+M_{6}\right) \Delta_{1}\left(I-\Delta_{1}\right)^{-1}\left|f_{m}\left(t, v_{m}(t)\right)\right| \\
& +M_{2} \Delta_{2}\left(I-\Delta_{2}\right)^{-1}\left\|g_{m}\left[v_{m}\right]\right\|,
\end{aligned}
$$

which is the same as (5.10).

In our next result we shall need the following:

CONDITION $c_{3}$ : Condition $c_{1}$ holds with (5.5) replaced by

$$
\left|f\left(t, v_{m}(t)\right)-f_{m}\left(t, v_{m}(t)\right)\right| \leq r_{3},
$$

where $r_{3}$ is a nonnegative $n \times 1$ vector.

CONDITION $c_{4}$ : Condition $c_{2}$ holds with (5.6) replaced by

$$
\left\|g\left[v_{m}\right]-g_{m}\left[v_{m}\right]\right\| \leq r_{4},
$$

where $r_{4}$ is a nonnegative $n \times 1$ vector.

Inequalities (5.13) and (5.14) correspond to the relative error in approximating $f$ and $g$ by $f_{m}$ and $g_{m}$. 
THEOREM 5.3. With respect to the boundary value problem (2.3), assume that there exists an approximate solution $\bar{x}(t) \in C(J)$, and

(i) conditions (i), (iii) of Theorem 5.2 are satisfied,

(ii) conditions $c_{3}$ and $c_{4}$ are satisfied,

(iii) $\rho\left(K_{1}\right)<1$ and $r_{5}=\left(I-K_{1}\right)^{-1}\left[\eta+2\left(M_{1}+M_{6}\right) r_{3}+2 M_{2} r_{4}\right] \leq r$.

Then

(1) all the conclusions (1)-(5) of Theorem 4.2 hold,

(2) the sequence $\left\{v_{m}(t)\right\}$ obtained from (5.4) remains in $\bar{S}\left(\bar{x}, r_{5}\right)$,

(3) conclusion (3) of Theorem 5.2 holds,

(4) the following error estimate holds:

$$
\left\|v_{m+1}-x^{*}\right\| \leq\left(I-K_{1}\right)^{-1}\left[K_{1}\left\|v_{m+1}-v_{m}\right\|+\left(M_{1}+M_{6}\right) r_{3}+M_{2} r_{4}\right] .
$$

PROOF. The proof is contained in Theorem 5.2.

Next we shall consider the following approximate Picard's scheme for Case 2.

$$
\begin{aligned}
v_{m+1}(t)= & \tilde{H}\left[f_{m}\left(t, v_{m}(t)\right)-A(t) v_{m}(t)\right]+\tilde{S}\left[l\left[v_{m}\right]-g_{m}\left[v_{m}\right]\right] \\
& +\tilde{S}_{1}\left[R_{3}\left(v_{m}-Q_{m} v_{m}\right)\right], \quad v_{0}(t)=x_{0}(t)=\bar{x}(t), \quad m=0,1, \ldots
\end{aligned}
$$

In (5.15), for each $m$ the function $f_{m}$ and the operator $g_{m}$ are assumed to be continuous. In addition, with respect to $f_{m}$ and $g_{m}$ we shall assume that the following conditions are satisfied.

CONDITION $c_{5}$ : For all $t \in C(J)$ and each $v_{m}(t)$ obtained from (5.15), the inequality (5.5) holds, where $\Delta_{1}$ is an $n \times n$ nonnegative matrix with $\rho\left(\Delta_{1}\right)<1$.

CONDITION $c_{6}$ : For each $v_{m}(t)$ obtained from (5.15), the inequality (5.6) holds, where $\Delta_{2}$ is an $n \times n$ nonnegative matrix with $\rho\left(\Delta_{2}\right)<1$.

THEOREM 5.4. With respect to the boundary value problem (2.3), assume that there exists an approximate solution $\bar{x}(t) \in C(J)$, and

(i) the conditions (i) and (ii) of Theorem 4.3 are satisfied,

(ii) the condition (iii) of Theorem 5.2 and conditions $c_{5}$ and $c_{6}$ are satisfied,

(iii) $\bar{K}_{2}=K_{2}+\left(M_{1}+\sigma M_{8}\right) \Delta_{1}\left(M_{3}+\|A(t)\|\right)+M_{2} \Delta_{2}\left(M_{4}+\|\ell\|\right) ; \rho\left(\bar{K}_{2}\right)<1$ and $\bar{r}_{2}=\left(I-K_{2}\right)^{-1}\left[\eta+2\left(M_{1}+\sigma M_{8}\right) \Delta_{1}\left(I-\Delta_{1}\right)^{-1}\left|f_{0}\left(t, v_{0}(t)\right)\right|+2 M_{2} \Delta_{2}(I-\right.$ $\left.\left.\Delta_{2}\right)^{-1}\left\|g_{0}\left[v_{0}\right]\right\|\right] \leq r$.

Then

(1) all the conclusions (1)-(5) of Theorem 4.3 hold,

(2) the sequence $\left\{v_{m}(t)\right\}$ obtained from (5.15) remains in $\bar{S}\left(\bar{x}, \bar{r}_{2}\right)$, 
(3) the sequence $\left\{v_{m}(t)\right\}$ converges to $x^{*}(t)$, the solution of (2.3) if and only if $\lim _{m \rightarrow \infty} c_{m}=0$, where

$$
\begin{aligned}
c_{m}= & \| v_{m+1}(t)-\tilde{H}\left[f\left(t, v_{m}(t)\right)-A(t) v_{m}(t)\right]-\tilde{S}\left[\ell\left[v_{m}\right]-g\left[v_{m}\right]\right] \\
& -\tilde{S}_{1}\left[R_{3}\left(v_{m}-Q v_{m}\right)\right] \|,
\end{aligned}
$$

(4) the following error estimate holds:

$$
\begin{aligned}
\left\|v_{m+1}-x^{*}\right\| \leq\left(I-K_{2}\right)^{-1}[ & K_{2}\left\|v_{m+1}-v_{m}\right\| \\
& +\left(M_{1}+\sigma M_{8}\right) \Delta_{1}\left(I-\Delta_{1}\right)^{-1}\left|f_{m}\left(t, v_{m}(t)\right)\right| \\
& \left.+M_{2} \Delta_{2}\left(I-\Delta_{2}\right)^{-1}\left\|g_{m}\left[v_{m}\right]\right\|\right] .
\end{aligned}
$$

Proof. The proof is similar to that of Theorem 5.2.

In our next result we shall need the following:

CONDITION $c_{7}$ : Condition $c_{5}$ holds with (5.5) replaced by (5.13).

CONDITION $c_{8}$ : Condition $c_{6}$ holds with (5.6) replaced by (5.14).

THEOREM 5.5. With respect to the boundary value problem (2.3), assume that there exists an approximate solution $\bar{x}(t) \in C(J)$, and

(i) condition (i) of Theorem 5.4 and (iii) of Theorem 5.2 are satisfied,

(ii) conditions $c_{7}$ and $c_{8}$ are satisfied,

(iii) $\rho\left(K_{2}\right)<1$ and $r_{5}=\left(I-K_{2}\right)^{-1}\left[\eta+2\left(M_{1}+\sigma M_{8}\right) r_{3}+2 M_{2} r_{4}\right] \leq r$.

Then

(1) all the conclusions (1)-(5) of Theorem 4.3 hold,

(2) the sequence $\left\{v_{m}(t)\right\}$ obtained from (5.15) remains in $\bar{S}\left(\bar{x}, r_{5}\right)$,

(3) conclusion (3) of Theorem 5.4 holds,

(4) the following error estimate holds:

$$
\left\|v_{m+1}-x^{*}\right\| \leq\left(I-K_{2}\right)^{-1}\left[K_{2}\left\|v_{m+1}-v_{m}\right\|+\left(M_{1}+\sigma M_{8}\right) r_{3}+M_{2} r_{4}\right] .
$$

PROOF. The proof is similar to that of Theorem 5.2.

\section{An example}

The following example illustrates the sharpness as well as the importance of our results. 
EXAMPLE 6.1. The boundary value problem

$$
\begin{aligned}
& u^{\prime \prime}+u+(u-t)^{3}=t+0.1 \\
& u(-1)=-0.9, \quad u(1)=1.1
\end{aligned}
$$

is due to Urabe [22], and has also appeared in [1, 5, 11, 25].

In system form (6.1) is the same as

$$
\begin{aligned}
& x_{1}^{\prime}=x_{2} \\
& x_{2}^{\prime}=-x_{1}-\left(x_{1}-t\right)^{3}+t+0.1 \\
& x_{1}(-1)=-0.9, \quad x_{1}(1)=1.1 .
\end{aligned}
$$

For (6.2) choose $\bar{x}(t)=(t+0.1,1)^{T}$

$$
A(t)=\left(\begin{array}{rr}
0 & 1 \\
-1 & 0
\end{array}\right)
$$

and

$$
l[x(t)]=\left(\begin{array}{ll}
1 & 0 \\
0 & 0
\end{array}\right)\left(\begin{array}{l}
x_{1}(-1) \\
x_{2}(-1)
\end{array}\right)+\left(\begin{array}{ll}
0 & 0 \\
1 & 0
\end{array}\right)\left(\begin{array}{l}
x_{1}(1) \\
x_{2}(1)
\end{array}\right) .
$$

As in [11], let $\epsilon=10^{-3}, \mu=1+\epsilon, \nu=1-\epsilon$,

$$
\tilde{\Phi}(t)=\left(\begin{array}{cc}
\mu \cos (1+t) & \mu \sin (1+t) \\
-\mu \sin (1+t) & \nu \cos (1+t)
\end{array}\right)
$$

and

$$
\hat{\Phi}(t)=\left(\begin{array}{cc}
\cos (1+t) & -v \sin (1+t) \\
\sin (1+t) & \mu \cos (1+t)
\end{array}\right)
$$

so that

$$
\begin{gathered}
\tilde{G}=\left(\begin{array}{cc}
\mu & 0 \\
\mu \cos 2 & \mu \sin 2
\end{array}\right) \\
\tilde{S}(t)=\frac{1}{\mu \sin 2}\left(\begin{array}{cc}
\mu \sin (1-t) & \mu \sin (1+t) \\
-C(t) & v \cos (1+t)
\end{array}\right)
\end{gathered}
$$

and

$$
\tilde{H}[\phi(t)]=\int_{-1}^{1} \tilde{H}(t, s) \phi(s) d s,
$$

where

$$
\begin{aligned}
& \sin 2 \tilde{H}(t, s)=\left(\begin{array}{cc}
\mu \sin (1-t) \cos (1+s) & -\mu \nu \sin (1-t) \sin (1+s) \\
-C(t) \cos (1+s) & \nu C(t) \sin (1+s)
\end{array}\right), \\
&=-\left(\begin{array}{cc}
\mu \sin (1+t) \cos (1-s) & \mu \sin (1+t) D(s) \\
\nu \cos (1+t) \cos (1-s) & \nu \cos (1+t) D(s)
\end{array}\right), \\
&-1 \leq t \leq s \leq 1 \leq 1
\end{aligned}
$$


and $C(t)=\cos (1-t)-\epsilon \cos (3+t), \quad D(t)=\sin (1-t)+\epsilon \sin (3+t)$.

Thus it follows that

$$
\begin{gathered}
\|\ell\|=\left(\begin{array}{ll}
1 & 0 \\
1 & 0
\end{array}\right) ; \quad\|\tilde{\Phi}\| \leq\left(\begin{array}{cc}
1.0010 & 0.9103 \\
0.9103 & 0.9990
\end{array}\right) ; \quad\left\|\tilde{G}^{-1}\right\| \leq\left(\begin{array}{ll}
0.9991 & 0.0000 \\
0.4573 & 1.0987
\end{array}\right) ; \\
\|\tilde{H}\| \leq\left\|\int_{-1}^{1}|\tilde{H}(t, s)| d s\right\| \leq \frac{1}{\sin 2}\left(\begin{array}{cc}
2 \mu & \mu(2 \sin 1-\sin 2+2 \epsilon \sin 2) \\
2 \mu & 2 \mu \sin ^{2} 1+2 \nu \epsilon
\end{array}\right) \\
\leq\left(\begin{array}{ll}
2.2017 & 0.8537 \\
2.2017 & 1.5612
\end{array}\right)=M_{1} \\
\|\tilde{S}\| \leq \frac{1}{\mu \sin 2}\left(\begin{array}{cc}
\mu & \mu \\
\mu & \nu
\end{array}\right) \leq\left(\begin{array}{ll}
1.0998 & 1.0998 \\
1.0998 & 1.0976
\end{array}\right)=M_{2} \\
\left\|\tilde{S}_{1}\right\| \leq\left(\begin{array}{ll}
3.1996 & 0 \\
2.1974 & 1
\end{array}\right)
\end{gathered}
$$

for $(t, x)$ such that $t \in[-1,1], x \in \bar{S}(\bar{x}, r), r=\left(r_{01}, r_{02}\right)$

$$
\begin{gathered}
\left\|f_{x}(t, x)-A(t)\right\|=\left\|\left(\begin{array}{cc}
0 & 0 \\
3\left(x_{1}(t)-t\right)^{2} & 0
\end{array}\right)\right\| \leq\left(\begin{array}{cc}
0 & 0 \\
3\left(0.1+r_{01}\right)^{2} & 0
\end{array}\right)=M_{3} ; \\
M_{4}=0 ; \\
M_{5}=\left(\begin{array}{cc}
0 & 1 \\
1+3\left(0.1+r_{01}\right)^{2} & 0
\end{array}\right) ; \\
\left\|\Gamma_{1}\right\| \leq 10^{-3}\left(\begin{array}{cc}
1 & 1.001 \\
1 & 0.001
\end{array}\right) ; \quad\|\Psi\| \leq 10^{-3}\left(\begin{array}{cc}
1 & 1.8186 \\
0 & 1.8323
\end{array}\right) ; \\
\left\|\Psi_{1}\right\| \leq 10^{-3}\left(\begin{array}{cc}
1.4162 & 0.9093 \\
0.9093 & 1.0000
\end{array}\right) ; \quad\left\|\Psi_{2}\right\| \leq 10^{-3}\left(\begin{array}{ll}
2.6557 & 0 \\
0.9103 & 1
\end{array}\right) ; \\
\sigma\left(\left\|\Psi_{2}\right\|\|A\|+\|\tilde{\Phi}\|\left\|\Psi_{1} A\right\|\right) \leq\left(\begin{array}{ll}
0.0037 & 0.0099 \\
0.0057 & 0.0063
\end{array}\right)=P_{1} ; \\
\sigma\left(\left\|\Psi_{2}\right\|+\|\tilde{\Phi}\|\left\|\Psi_{1}\right\|\right) \leq\left(\begin{array}{ll}
0.0099 & 0.0037 \\
0.0063 & 0.0057
\end{array}\right) \leq P_{2} ; \\
\left\|\tilde{S}_{1}\right\| P_{2} \leq\left(\begin{array}{ll}
0.0317 & 0.0119 \\
0.0281 & 0.0139
\end{array}\right)=M_{6} ; \quad \text { (cf. Remark 4.1); } \\
\left\|\tilde{S}_{1} \Psi_{2}\right\| \leq\left(\begin{array}{ll}
0.0085 & 0.0000 \\
0.0068 & 0.0010
\end{array}\right)=M_{7} ;
\end{gathered}
$$




$$
\begin{aligned}
& e^{\sigma\|A\|} \leq\left(\begin{array}{cc}
\cosh 2 & \sinh 2 \\
\sinh 2 & \cosh 2
\end{array}\right) \leq\left(\begin{array}{ll}
3.7622 & 3.6269 \\
3.6269 & 3.7622
\end{array}\right) \\
& \left\|\tilde{G}_{1}\right\|\|\ell\| \exp (\sigma\|A\|)\|\Psi\| \leq\left(\begin{array}{ll}
0.0038 & 0.0135 \\
0.0059 & 0.0210
\end{array}\right)=M \text {; } \\
& \left\|\tilde{S}_{1} \Psi_{2}\right\|+\left\|\tilde{S}_{1}\right\| P_{1} \leq\left(\begin{array}{ll}
0.0204 & 0.0317 \\
0.0207 & 0.0291
\end{array}\right)=\bar{M} \\
& \left\|\tilde{S}_{1} \tilde{E}_{1}[Q \bar{x}]+\tilde{S}[g[\bar{x}]]\right\| \leq 10^{-3}\left(\begin{array}{l}
5.8246 \\
6.0002
\end{array}\right)=\eta_{1} \\
& K_{1}=\left(\begin{array}{ll}
0.0204+2.5968\left(0.1+r_{01}\right)^{2} & 0.0317 \\
0.0207+4.7253\left(0.1+r_{01}\right)^{2} & 0.0291
\end{array}\right) \text {; } \\
& \sigma\left\|A_{1}-\Gamma A\right\| \leq 0.002\left(\begin{array}{ll}
0.0021 & 1.0021 \\
2.0010 & 2.0041
\end{array}\right)=N \\
& \left(I-\left\|\Gamma_{1}\right\|\right)^{-1} \leq\left(\begin{array}{ll}
1.0011 & 0.0011 \\
0.0011 & 1.0001
\end{array}\right)=Z \text {; } \\
& \left\|\Gamma A+\tilde{\Phi} \hat{\Phi}^{\prime}\right\| \leq 10^{-3}\left(\begin{array}{ll}
1.001 & 1.001 \\
1.001 & 2.000
\end{array}\right) \\
& \|\tilde{H}\| Z\left\|\Gamma A+\tilde{\Phi} \hat{\Phi}^{\prime}\right\|+\left\|\tilde{S}_{1}\right\|\left\|\Gamma_{1}\right\| \leq 10^{-3}\left(\begin{array}{ll}
6.2640 & 7.1225 \\
6.9708 & 7.5362
\end{array}\right)=P_{3}=M_{8} \\
& \text { (cf. (4.10), (4.12), (4.13)); } \\
& K_{2}=\left(\begin{array}{ll}
0.0206+2.6039\left(0.1+r_{01}\right)^{2} & 0.0197 \\
0.0221+4.7289\left(0.1+r_{01}\right)^{2} & 0.0215
\end{array}\right) \text {. }
\end{aligned}
$$

To apply Theorem 4.2 we note that from the above computation, $\rho(M)<1$ and $\rho(\bar{M})<1$, and therefore in view of Remark 4.1, conditions of Lemma 3.3 are satisfied, and in conclusion $\tilde{L}^{-1}$ is invertible. Next, $\rho\left(K_{1}\right)<1$ if and only if

$$
0 \leq r_{01}<0.4965
$$

Further, the condition $\left(I-K_{1}\right)^{-1} \eta_{1} \leq r$ implies that

$$
6.35204 \times 10^{-3} \leq r_{01} \leq 0.492783
$$

and

$$
r_{02} \geq \frac{10^{-3}\left(5.9984+11.9417\left(0.1+r_{01}\right)^{2}\right)}{0.9504-2.671025\left(0.1+r_{01}\right)^{2}}
$$


For $r_{01}=6.4 \times 10^{-3}$, both (6.5) and (6.6) are satisfied. Also, for this value of $r_{01}$ from (6.3) and (6.7), we have

$$
K_{1}=\left(\begin{array}{ll}
0.0498 & 0.0317 \\
0.0742 & 0.0291
\end{array}\right) \quad \text { and } \quad r_{02} \leq 6.66578 \times 10^{-3} .
$$

In conclusion, the following hold:

(1) there exists a solution $x^{*}(t)$ of $(6.2)$ in $\bar{S}(\bar{x}, r)=\left\{\left(x_{1}, x_{2}\right):\left|x_{1}-(t+0.1)\right|\right.$ $\left.\leq 6.4 \times 10^{-3},\left|x_{2}-1\right| \leq 6.66578 \times 10^{-3}\right\}$,

(2) $x^{*}(t)$ is the unique solution of $(6.2)$ in $\bar{S}(\bar{x}, r)=\left\{\left(x_{1}, x_{2}\right):\left|x_{1}-(t+0.1)\right|\right.$ $\leq 0.492783\}$,

(3) the sequence $\left\{u_{m}(t)\right\}$ generated by (4.2) for the problem (6.2) remains in $\bar{S}(\bar{x}, r)=\left\{\left(x_{1}, x_{2}\right):\left|x_{1}-(t+0.1)\right| \leq 6.4 \times 10^{-3},\left|x_{2}-1\right| \leq 6.66578\right.$ $\left.\times 10^{-3}\right\}$ and converges to $x^{*}(t)$,

(4) the following error estimate is valid:

$$
\left|x^{*}(t)-u_{m}(t)\right| \leq 10^{-3}\left(\begin{array}{ll}
0.0498 & 0.0317 \\
0.0742 & 0.0291
\end{array}\right)^{m}\left(\begin{array}{l}
6.40000 \\
6.66578
\end{array}\right) .
$$

To apply Theorem 4.3 from the above computation, it is clear that $\rho(N)<1$, and therefore conditions of Lemma 3.5 are satisfied, and in conclusion $\tilde{L}^{-1}$ is invertible. Next, $\rho\left(K_{2}\right)<1$ if and only if

$$
0 \leq r_{01}<0.502243 .
$$

Further, the condition $\left(I-K_{2}\right)^{-1} \eta_{1} \leq r$ implies that

$$
6.26839 \times 10^{-3} \leq r_{01} \leq 0.498563
$$

and

$$
r_{02} \geq \frac{10^{-3}\left(6.005+11.9200\left(0.1+r_{01}\right)^{2}\right)}{0.9579-2.6411\left(0.1+r_{01}\right)^{2}}
$$

Once again for $r_{01}=6.4 \times 10^{-3}$ both (6.8) and (6.9) are satisfied. Also, for this value of $r_{01}$ from (6.4) and (6.10), we have

$$
K_{2}=\left(\begin{array}{ll}
0.0501 & 0.0197 \\
0.0757 & 0.0215
\end{array}\right) \quad \text { and } \quad r_{02} \geq 6.61632 \times 10^{-3} .
$$

In conclusion, the following hold

(1) there exists a solution $x^{*}(t)$ of $(6.2)$ in $\bar{S}(\bar{x}, r)=\left\{\left(x_{1}, x_{2}\right):\left|x_{1}-(t+0.1)\right|\right.$ $\left.\leq 6.4 \times 10^{-3},\left|x_{2}-1\right| \leq 6.61632 \times 10^{-3}\right\}$,

(2) $x^{*}(t)$ is the unique solution of $(6.2)$ in $\bar{S}(\bar{x}, r)=\left\{\left(x_{1}, x_{2}\right):\left|x_{1}-(t+0.1)\right|\right.$ $\leq 0.498563$, 
(3) the sequence $\left\{u_{m}(t)\right\}$ generated by (4.2) for the problem (6.2) remains in $\bar{S}(\bar{x}, r)=\left\{\left(x_{1}, x_{2}\right):\left|x_{1}-(t+0.1)\right| \leq 6.4 \times 10^{-3},\left|x_{2}-1\right| \leq 6.61632\right.$ $\left.\times 10^{-3}\right\}$ and converges to $x^{*}(t)$,

(4) the following error estimate is valid:

$$
\left|x^{*}(t)-u_{m}(t)\right| \leq 10^{-3}\left(\begin{array}{ll}
0.0501 & 0.0197 \\
0.0757 & 0.0215
\end{array}\right)^{m}\left(\begin{array}{l}
6.40000 \\
6.61632
\end{array}\right) .
$$

\section{Acknowledgements}

The authors are grateful to referees for their comments on the first draft of this paper.

\section{References}

[1] R. P. Agarwal, "Contraction and approximate contraction with an application to multi-point boundary value problems", J. Comp. Appl. Math. 9 (1983) 315-325.

[2] R. P. Agarwal, "Component-wise convergence of iterative methods for non-linear Volterra integrodifferential systems with nonlinear boundary conditions", Math. Phyl. Sci. 18 (1984) 291-322.

[3] R. P. Agarwal, "On Urabe's application of Newton's method to nonlinear boundary value problems", Arch. Math. (Bmö) 20 (1984) 113-124.

[4] R. P. Agarwal, "Computational methods for discrete boundary value problems", Appl. Math.Comp. 18 (1986) $15-41$.

[5] R. P. Agarwal, "Component-wise convergence of quasilinear method for nonlinear boundary value problems", Hiroshima Math. J. 22 (1992) 525-541.

[6] R. P. Agarwal, "Computational methods for discrete boundary value problems II", J. Math. Anal. Appl. 166 (1992) 540-562.

[7] R. P. Agarwal and J. Vosmanskỳ, "Necessary and sufficient conditions for the convergence of approximate Picard's iterates for nonlinear boundary value problems", Arch. Math. (Brnö) 21 (1985) 171-176.

[8] S. R. Bernfeld and V. Lakshmikantham, An introduction to nonlinear boundary value problems (Academic Press, New York, 1974).

[9] M. Fujii, "An a posteriori error estimation of the numerical solution by step-by-step methods for systems of ordinary differential equations", Bull. Fukoka Univ. Ed. III 23 (1973) 35-44.

[10] M. Fujii and Y. Hayashi, "Numerical solutions to problems of the least squares type for ordinary differential equations", Hiroshima Math. J. 13 (1983) 477-499.

[11] Y. Hayashi, "On a posteriori error estimation in the numerical solution of systems of ordinary differential equations", Hiroshima Math. J. 9 (1979) 201-243.

[12] N. S. Kurpel', Projection-iterative methods for solutions of operator equations, Translations of Math. Monographs (Amer. Math. Soc., Providence, Rhode Island, 1976).

[13] T. Mitsui, "The initial-value adjusting method for problems of the least square type of ordinary differential equations", Publ. RIMS - Kyoto Univ. 16 (1980) 785-810.

[14] T. Mitsui, "Iterative approximation methods for non-linear boundary value problems of ODE", Lecture Notes in Num. Appl, Anal. (The Newton Method and Related Topics) 3 (1981) 105-125. 
[15] T. Ojika, "On quadratic convergence of the initial estimates and iterative methods for nonlinear multipoint boundary value problems", J. Math. Anal. Appl. 73 (1980) 192-203.

[16] T. Ojika and T. Kasue, "Initial-value adjusting method for the solution of nonlinear multipoint boundary-value problems", J. Math. Anal. Appl. 69 (1979) 359-371.

[17] T. Ojika and W. Welsh, "A numerical method for the solution of multi-point problems for ordinary differential equations with integral constraints", J. Math. Anal. Appl. 72 (1979) 500-511.

[18] J. Schröder, Operator inequalities (Academic Press, New York, 1980).

[19] H. Shintani and Y. Hayashi, "A posteriori error estimates and iterative methods in the numerical solution of systems of ordinary differential equations", Hiroshima Math. J. 8 (1978) 101-121.

[20] Radha Shridharan and R. P. Agarwal, "Stationary and nonstationary iterative methods for nonlinear boundary value problems", Math. Comp. Modelling 18 (1993) 43-62.

[21] K. L. Teo, C. J. Goh and K. H. Wong, A unified computational approach to optimal control problems (Longman Scientific and Technical, Essex, England, 1991).

[22] M. Urabe, "An existence theorem for multi-point boundary value problems", Funkcialaj Ekvacioj 9 (1966) 43-60.

[23] M. Urabe, "The Newton method and its application to boundary value problems with nonlinear boundary conditions", in Proc. US - Japan Seminar on Differential and Functional Equations (Benjamin, 1967) 383-410.

[24] M. Urabe, "On the Newton method to solve problems of the least square type for ordinary differential equations", Memoirs of the Faculty of Sci. Kyushu Univ. Ser. A 29 (1975) 173-183.

[25] T. Yamamoto, "An existence theorem of solution to boundary value problems and its applications to error estimates”, Math. Japonica 27 (1982) 301-318. 